\title{
Circadian rhythm of autonomic activity in non diabetic offsprings of type 2 diabetic patients
}

\author{
A Fiorentini ${ }^{* 1}$, A Perciaccante ${ }^{1}$, A Paris ${ }^{1}$, P Serra $^{1}$ and L Tubani ${ }^{2}$
}

\author{
Address: ${ }^{1}$ III Clinica Medica, Department of Clinical Medicine, University "La Sapienza", Rome, Italy and ${ }^{2}$ Medicina Interna E, Department of \\ Clinical Medicine, University "La Sapienza", Rome, Italy \\ Email: A Fiorentini* - fiorentini-a@libero.it; A Perciaccante - antonioperciaccante@libero.it; A Paris - alberto.paris@uniroma1.it; \\ P Serra - pietroserra@uniroma1.it; L Tubani - tubani@libero.it \\ * Corresponding author
}

Published: 0I October 2005

Cardiovascular Diabetology 2005, 4:15 doi:10.1/86/1475-2840-4-15

This article is available from: http://www.cardiab.com/content/4/I/I5

(C) 2005 Fiorentini et al; licensee BioMed Central Ltd.

This is an Open Access article distributed under the terms of the Creative Commons Attribution License (http://creativecommons.org/licenses/by/2.0), which permits unrestricted use, distribution, and reproduction in any medium, provided the original work is properly cited.
Received: 0I August 2005

Accepted: 01 October 2005

\begin{abstract}
The aim of the present study was to evaluate, by heart rate variability (HRV) with 24-hours ECG Holter (HRV), the circadian autonomic activity in offspring of type 2 diabetic subjects and the relation with insulin-resistance. METHODS: 50 Caucasian offsprings of type 2 diabetic subjects were divided in two groups: insulin-resistant offsprings (IR) and non insulin-resistant offsprings (NIR). Autonomic nervous activity was studied by HRV. Time domain and spectral analysis (low frequency, LF, and high frequency, HF, provide markers of sympathetic and parasympathetic modulation when assessed in normalized units) were evaluated. RESULTS. Time domain showed a reduction of total SDNN in IR $(p<0.00 I)$ and NIR ( $p$ 0.047) versus controls. Spectral analysis showed a total and night LF higher in IR and NIR than in control group (all $\mathrm{p}<0.00 \mathrm{I}$ ). CONCLUSION. In frequency domain, the analysis of sympathetic (LF) and parasympathetic (HF) component evidenced an association between the offspring of type 2 diabetic subjects and a sympathetic overactivity. A global reduction and alteration of circadian rhythm of autonomic activity are present in offspring of type 2 diabetic patients with and without insulin resistance. The data of our study suggested that an autonomic impairment is associated with the familiarity for type 2 diabetes independently to insulin resistance and that an impairment of autonomic system activity could precede the insulin resistance.
\end{abstract}

\section{Background}

Sympathetic and parasympathetic components of neurovegetative system regulate cardiac activity. Spectral analysis of heart rate variability (HRV) is a non invasive metodic used to assess cardiac autonomic activity. The autonomic activities can be assessed in HRV by the relative distribution (evaluated in normalized units) of low frequency (LF), as an index of sympathetic modulation and of high frequency (HF), as an index of parasympathetic modulation. The analysis of HRV has been used for evaluate the autonomic cardiac activity in numerous pathophisyologic conditions [1-9]: an impaired heart rate variation is a marker of autonomic neuropathy $[10,11]$ as in diabetic subjects [12-16]. 
Table I: Clinical characteristics for each study-group of offsprings and controls.

\begin{tabular}{lcccc}
\hline & IR & NIR & Controls & P Value \\
\hline Sex (M/F) & $16 / 9$ & $18 / 7$ & $16 / 9$ & \\
Age (years) & $49,12 \pm 6,68$ & $49,80 \pm 6,53$ & $53,69 \pm 3,72$ & 0,190 \\
BMI (kg/m2) & $25.41 \pm 4.67$ & $25.50 \pm 3.69$ & $25.09 \pm 2.23$ & 0,120 \\
Glycemia '0 (mg/dl) & $93,375 \pm 11,06$ & $99,66 \pm 1,30$ & $94,00 \pm 6,15$ & 0,095 \\
Glycemia 'I 20 (mg/dl) & $97,62 \pm 17,45$ & $90,00 \pm 26,60$ & $92,66 \pm 18,22$ & 0,095 \\
Fasting plasma insulin (uU/mI) & $17,95 \pm 6,35$ & $7,66 \pm 0,50$ & $7,16 \pm 0,21$ & 0,000 \\
HOMA index & $4,41 \pm 0,80$ & $1.83 \pm 0,12$ & $1,45 \pm 0,25$ & 0,013 \\
SP (mmHg) & $123,20 \pm 3,70$ & $122,60 \pm 6,35$ & $120.20 \pm 7.09$ & 0,014 \\
DP(mmHg) & $80,00 \pm 3,16$ & $79,80 \pm 3,56$ & $78.80 \pm 4.90$ & 0,011 \\
HR (bpm) & $74.75 \pm 4.57$ & $70.25 \pm 4.11$ & $68.50 \pm 5.92$ &
\end{tabular}

SP: systolic pressure, DP: diastolic pressure, HR: heart rate, BMI: body mass index, HOMA-I: the homeostasis model assessment-index, IR: insulin resistant offsprings of type 2 diabetic patients, NIR: non insulin resistant offsprings of type 2 diabetic patients.

Several studies have showed a sympathetic overactivity also in non diabetic insulin resistant group [17-19]. However, little is known about the association between the familiarity for type 2 diabetes mellitus and the autonomic activity. An increased LF/ HF ratio (low frequency/high frequency ratio) has identified in only insulin resistant offsprings of type 2 diabetic subjects [20]. De Angelis et al [21] has showed this condition at rest but not under stimulated conditions. Laitinen et al [22] has identified a sympathetic overactivity during acute hyperinsulinemia both in insulin resistant and non insulin resistant offsprings of type 2 diabetic subjects.

The aim of the present study was to evaluate, by heart rate variability with 24-hours ECG Holter registration, the autonomic activity and circadian autonomic rhythm in offspring of type 2 diabetic subjects and to evaluate the possible impact of sympathetic activity on insulin resistance. We tested the hypothesis that in non insulin resistant offsprings of type 2 diabetic subjects an alteration of circadian rhythm of autonomic activity are present and that the impairment of autonomic system activity could precede the insulin resistance.

\section{Subjects and methods}

70 consecutive caucasian offsprings of type 2 diabetic subjects, admitted to our department, were screened. In all subjects, after an overnight fast, oral glucose tolerance tests (OGTTs) was performed: samples blood for glucose and plasma insulin were collected before and $2 \mathrm{~h}$ after a glucose load consisting of $75 \mathrm{~g}$ glucose anhydrate in 300 $\mathrm{ml}$ of water ingested over the course of $5 \mathrm{~min}$. Also, fasting plasma insulin was measured to evaluate the insulinresistance by the homeostasis model assessment-index (HOMA-I). Among them, 50 caucasian subjects (age: $47.71 \pm 9.96$ years, 32 men and 18 women), with normal OGTTs, were admitted in this study and were divided in two groups: offsprings with insulin resistance and offsprings without insulin resistance:

Subjects with hypertension [23], diabetes mellitus, impaired fasting glycemia, impaired glucose tolerance [24], obesity, dyslipidemia, cardiac arrhythmias, microalbuminuria and with drug treatment or diseases that could potentially disturbs carbohydrate metabolism (glucocorticoids, furosemide, beta-blockers, etc.) and cardiac autonomic activity (beta-blockers, anti-arrhythmics, ACEinhibitors) were excluded.

The control group consisted of 25 sex and age matched healthy non insulin resistant subjects with normal OGTTs and without familiarity for type 2 diabetes mellitus.

Height, weight and body circumferences were measured on all subjects. Body mass index (BMI, $\mathrm{kg} / \mathrm{m}^{2}$ ) was calculated as weight divided by height squared. Waist-to-hip ratio (WHR) was defined as waist circumference divided by hip circumference.

Informed consent was obtained from all participants; all the investigations were performed in accordance with the participants of the Declaration of Helsinki.

\section{Insulin resistance}

The insulin-resistance was evaluated by the homeostasis model assessment index (HOMA-I) [25-28]. The HOMAI was calculated by the formula: fasting plasma glucose $(\mathrm{mmol} / \mathrm{L}) \times$ fasting plasma insulin $(\mu \mathrm{U} / \mathrm{ml}) / 22,5$, as described by Matthews and coworkers [29]. Insulin-resistance was defined as the third and fourth quartiles of HOMA-I. 
Table 2: Means $(95 \% \mathrm{Cl})$ RR intervals in the different time periods for each offspring group and controls.

\begin{tabular}{llll}
\hline R-R (msec) & IR & NIR & Controls \\
\hline Night $(0-6$ am) & $895,39(821,61-969,17)$ & $873,36(781,09-965,64)$ & $1126,75(1060,44-1193,05)$ \\
morning (7-12 am) & $792,44(741,58-843,29)$ & $752,26(719,55-784,98)$ & $870,29(805,43-935,15)$ \\
afternoon (I-6 pm) & $690,28(658,75-721,82)$ & $689,16(638,25-740,07)$ & $749,54(615,63-883,46)$ \\
evening (7-II pm) & $734,37705,02-763,72)$ & $596,87(360,36-833,39)$ & $910,82(808,78-1012,87)$
\end{tabular}

Value are means $(95 \% \mathrm{CI})$, IR: insulin resistant offsprings of type 2 diabetic patients, NIR: non insulin resistant offsprings of type 2 diabetic patients, RR: normal-to-normal R-R interval (ms)

The index subject were subdivide into two groups based on HOMA-I: 1) group of insulin-resistant offsprings (IR); 2) group of non insulin-resistant offsprings (NIR).

\section{HRV assessment}

Autonomic nervous activity was evaluated by heart rate variability (HRV) analysis during 24-hour ECG recording. All Holter recordings were performed using a three-channel recorder. Autonomic nervous activity was analysed following the recommendations of the Task Force of the European Society of Cardiology and the North American Society of Pacing and Electrophysiology [30]. Spectral estimates of R-R interval were obtained from stationary regions free of ectopic beats and technical artefacts. The standard deviation of normal-to-normal RR intervals (SDNN) (ms) and the square root of the mean of the sum of the squares of differences between adjacent $\mathrm{NN}$ intervals (RMS-SD), correlated with parasympathetic system, were calculated and were divided in two periods: night $(0$ am $-6 a m)$ and day $(7 \mathrm{am}-9 \mathrm{pm})$. Fast Fourier Transform was used to obtain power spectral estimates of HRV. Total power in the frequency range $(0-0.40 \mathrm{~Hz})$ was divided into low frequency (LF: $0.04-0.15 \mathrm{~Hz}$, modulated by sympathetic system) and high frequency (HF: $0.15-0.40 \mathrm{~Hz}$, modulated by parasympathetic system). The power of LF and HF components was considered in normalized units (n.u.). Subjects were analysed for 24 hours, at 10 minutes interval. Artificial data and arrhythmic were excluded. The day was divided in four periods: night (0 am - $6 \mathrm{am})$, morning (7 am - $12 \mathrm{am})$, afternoon $(1 \mathrm{pm}-6 \mathrm{pm})$, evening $(7 \mathrm{pm}-11 \mathrm{pm})$. Data analyses were performed with software Del Mar Avionics Accuplus 363, Irvine California, USA.

\section{Statistical analysis}

All analysis were done with SPSS 12.0 (SPSS Inc., Chicago, IL, USA) for Windows XP. Data are presented as means + SD. For data with multiple time points, variables were analysed by the general linear model ANOVA and simple regression analyses were carried out by standard techniques $95 \%$ confidence intervals (CT) were calculated for regression coefficient. Means values were considered significant at $\mathrm{p}<0.05$.

\section{Results \\ Clinical characteristics}

The groups had not statistically significant difference in age, sex and anthropometrics parameters (i.e. BMI, waist and hip circumference, waist to hip ratio, table 1).

\section{Heart rate variability}

Tables 2 and 3 show the means of autonomic function measures for each group.

\section{Time domain}

Time domain analysis of HRV showed a reduction statistically significant of total SDNN in IR $(\mathrm{p}<0.001)$ and NIR (p 0.047) groups (IR: $86.10 \pm 35.96 \mathrm{~ms}$, NIR: $119.70 \pm$ $28.22 \mathrm{~ms})$ versus control group $(131.90 \pm 29.52 \mathrm{~ms})$ (fig. 1). Total SDNN were reduced in IR group when compared with (NIR) NIR group ( $p$ 0.003). These results showed a total activity reduction of autonomic system in insulin resistant and non insulin resistant offsprings of type 2 diabetic patients. The autonomic activity reduction is major in IR group than NIR group.

RMS-SD in night time not increased in IR group. Therefore insulin-resistance was associated with alteration of circadian rhythm of parasympathetic component (at the night).

\section{Frequency domain}

Frequency domain analysis of HRV showed a total LF higher (all p < 0.001) in IR $(70.88 \pm 6.2$ n.u.) and NIR $(67.59 \pm 4.46$ n.u.) groups than in control group (55.46 \pm 7.49 n.u.). No difference was noticed between IR and NIR groups (p: 0.157$)$. HF were lower in IR $(23.63 \pm 6.67$ n.u $)$ and NIR $(27.09 \pm 3.74$ n.u.) than in control group (37.65 $\pm 7.53)$ (all p $<0.001)$, while not statistically significant difference were between the groups of offsprings type 2 diabetic patients, $\mathrm{p}: 0.127$. LF/HF total is higher in IR $(3.34 \pm 1.37)$ and NIR $(3.72 \pm 0.72)$ groups versus control group $(2.44 \pm 1.09)$, respectively p 0.016 and p 0.002 .

\section{Night (0 am - $6 a m)$}

LF value are higher in IR $(67.80 \pm 9.70$ n.u. $)$ and NIR $(59.60 \pm 1.75$ n.u.) groups than in control groups (35.21 
Table 3: Autonomic function measures for each offspring group and controls.

\begin{tabular}{ccccc}
\hline & IR & NIR & Controls & P value \\
\hline SDNN (msec) & $86.10 \pm 35.96$ & $119.70 \pm 28.22$ & $131.90 \pm 29.52$ & 0,000 \\
SDNN day (msec) & $105.21 \pm 28.37$ & $128.77 \pm 30.60$ & $120.29 \pm 26.97$ & 0,062 \\
SDNN night (msec) & $118.99 \pm 46.16$ & $112.40 \pm 7.54$ & $119.57 \pm 38.25$ & 0,822 \\
RMS-SD (msec) & $43.15 \pm 20.60$ & $42.63 \pm 9.18$ & $36.75 \pm 10.59$ & 0,331 \\
RMS-SD day (msec) & $41.58 \pm 18.64$ & $34.77 \pm 5.75$ & $31.37 \pm 9.07$ & 0,050 \\
RMS-SD night (msec) & $47.47 \pm 28.80$ & $55.70 \pm 18.46$ & $47.47 \pm 10.60$ & 0,485 \\
LF u.n. & $70.88 \pm 6.20$ & $67.59 \pm 4.46$ & $55.46 \pm 7.49$ & 0,000 \\
HF u.n. & $23.63 \pm 6.67$ & $27.09 \pm 3.74$ & $37.65 \pm 7.53$ & 0,000 \\
LF/HF & $3.34 \pm 1.37$ & $3.72 \pm 0.72$ & $2.44 \pm 1.09$ & 0,004 \\
LF night u.n. & $3.34 \pm 1.37$ & $3.72 \pm 0.72$ & $2.44 \pm 1.09$ & 0,000 \\
HF night u.n. & $25.72 \pm 8.19$ & $36.53 \pm 3.17$ & $58.62 \pm 6.59$ & $0.79 \pm 0.48$ \\
LF/HF night & $3.06 \pm 1.49$ & $2.21 \pm 0.54$ & $69.54 \pm 8.36$ & 0,000 \\
LF u.n. morning & $73.45 \pm 9.61$ & $69.04 \pm 7.41$ & $23.05 \pm 7.13$ & 0,298 \\
HF u.n. morning & $22.12 \pm 7.17$ & $24.30 \pm 8.52$ & $3.70 \pm 1.72$ & 0,765 \\
LF/HF morning & $4.15 \pm 2.80$ & $5.69 \pm 3.11$ & 0,122 \\
LF u.n. afternoon & $70.59 \pm 10.62$ & $70.06 \pm 4.26$ & $22.30 \pm 5.09$ & 0,979 \\
HF u.n. afternoon & $23.21 \pm 10.25$ & $21.42 \pm 3.79$ & $3.67 \pm 1.30$ & 0,794 \\
LF/HF afternoon & $4.65 \pm 1.71$ & $3.74 \pm 0.46$ & $56.75 \pm 7.75$ & 0,647 \\
LF u.n. evening & $71.90 \pm 10.70$ & $71.90 \pm 10.70$ & $35.75 \pm 9.14$ & 0,000 \\
HF u.n. evening & $23.52 \pm 6.28$ & $24.28 \pm 9.08$ & $2.00 \pm 0.88$ & 0,001 \\
LF/HF evening & $3.30 \pm 1.00$ & $4.12 \pm 2.21$ & & 0,009 \\
\hline
\end{tabular}

Value are means \pm SD, IR: insulin resistant offsprings of type 2 diabetic patients, NIR: non insulin resistant offsprings of type 2 diabetic patients, SDNN: standard deviation of all sinus rhytm RR intervals, RMS SD: the square root of the mean of the sum of the squares of differencies between adjacent NN intervals, LF: low frequence, HF: high frequence, hours of Holter registration: night time $=0-6$ am, morning $=7-12$ am, afternoon $=$ $1-6 \mathrm{pm}$, evening $=7-\mathrm{II} \mathrm{pm}$. P Value is between groups.

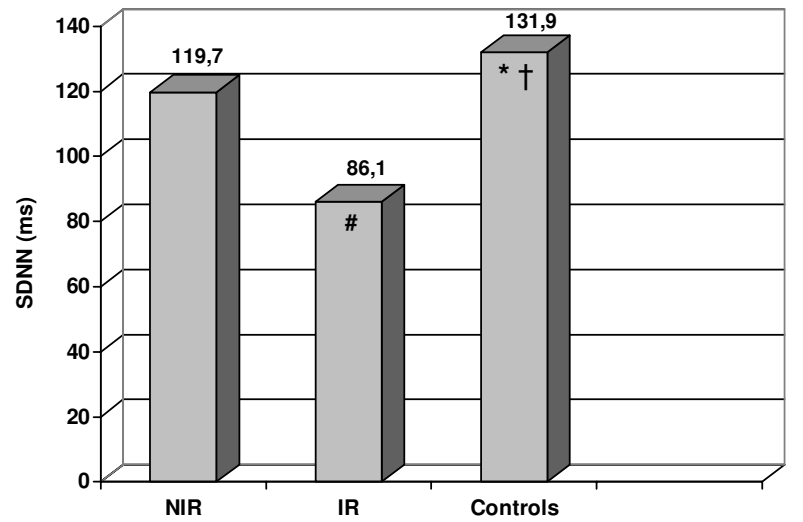

Figure I

SDNN value in offsprings of type 2 diabetic patients and controls. SDNN: standard deviation of all sinus rhythm RR intervals, IR: insulin resistant offsprings of type 2 diabetic patients, NIR: non insulin resistant offsprings of type 2 diabetic patients. \#p:0.003IR vs NIR; ${ }^{*}$ : 0.047 controls vs NIR and $\dagger p: 0.001$ controls vs IR \pm 5.97 ) (all $p<0.001)$ and are higher in IR group when compared with NIR group, p 0.001). HF are lower in IR $(25.72 \pm 8.19$ n.u. $)$ and NIR group $(36.53 \pm 3.17)$ than in control groups $(58.62 \pm 6.59)$ (all $\mathrm{p}<0.001$ vs controls).

Morning (7 am - $12 \mathrm{am})$

LF and HF had not statistically difference between IR (LF: $73.45 \pm 9.61$ n.u.; HF n.u.: $22.12 \pm 7.17$ n.u.), NIR (LF: $69.04 \pm 7.41$ n.u.; HF: $24.30 \pm 8.52$ n.u) and control groups (LF: $69.54 \pm 8.36$ n.u.; HF: $23.05 \pm 7.13$ n.u.).

Afternoon (I pm - 6 pm)

LF, HF and LF/HF had not statistically difference between IR (LF: $70.59 \pm 10.62$ n.u.; HF: $23.21 \pm 10.25$ n.u.; LF/HF: $4.65 \pm 1.71$ ), NIR (LF: $70.06 \pm 4.26$ n.u.; HF: $21.42 \pm 3.79$ n.u.; LF/HF: $3.74 \pm 0.46$ ) and control groups (LF: $70.09 \pm$ 6.62 n.u.; HF: $22.30 \pm 5.09$ n.u.; LF/HF: $3.67 \pm 1.30$ ).

\section{Evening $(7 \mathrm{pm}-1 / \mathrm{pm})$}

LF were higher in IR (71.90 \pm 10.70 n.u.) and NIR (71.90 \pm 10.70 n.u.) groups than control group $(56.75 \pm 7.75$ n.u.) (all p < 0.001). HF were lower in IR $(23.52 \pm 6.28$ n.u.) and NIR $(24.28 \pm 9.08$ n.u. $)$ groups than control group $(35.75 \pm 9.14$ n.u. $)$, respectively p: 0.001 and $p$ : 


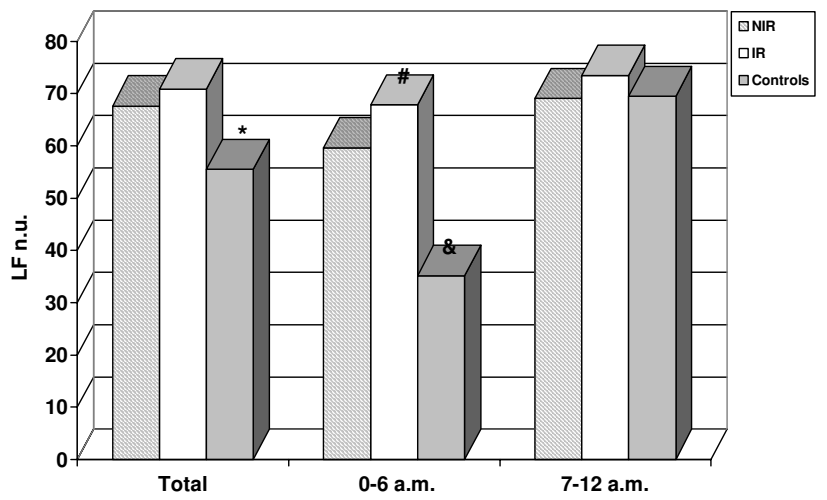

Figure 2

LF value in offsprings of type 2 diabetic patients and control. IR: insulin resistant offsprings of type 2 diabetic patients, NIR: non insulin resistant offsprings of type 2 diabetic patients, LF: low frequency, hours of Holter registration: $0-6 \mathrm{am}=$ night time, $7-12 \mathrm{am}=$ morning. ${ }^{*} \mathrm{p}<0.00 \mathrm{I}$ NIR and IR vs controls, \#: p:0.00I IR vs NIR; \& $p<0.00$ I controlsvs NIR and IR.

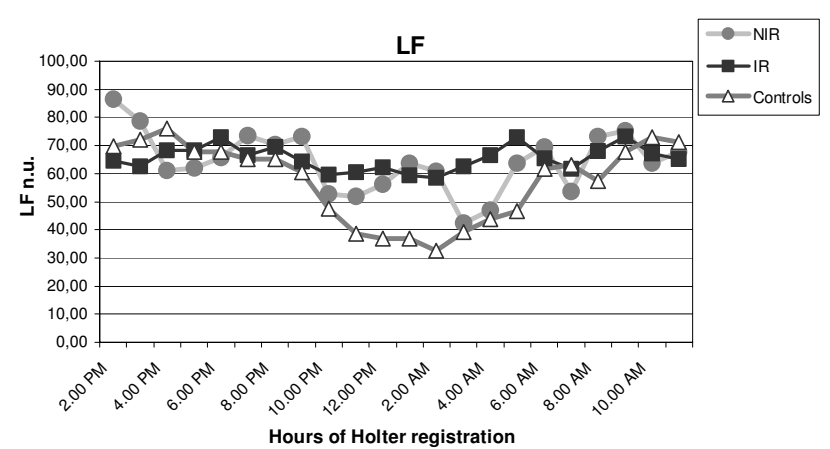

Figure 3

Circadian variation of LF in IR, NIR and in control group. Gray circles: group of non insulin resistant offsprings of type 2 diabetic patients; black squares: group of insulin resistant offsprings of type 2 diabetic patients; white triangles: control group; IR: insulin resistant offsprings of type 2 diabetic patients, NIR: non insulin resistant offsprings of type 2 diabetic patients, LF: low frequency.

0.002. $\mathrm{LF} / \mathrm{HF}$ is higher in IR $(3.30 \pm 1.00)$ and NIR $(4.12$ $\pm 2.21)$ groups than control group $(2.00 \pm 0.88)$ (respectively p: 0.04 and p: 0.002).

\section{Discussion}

The data of our study suggested that an autonomic impairment is associated with the familiarity for type 2 diabetes

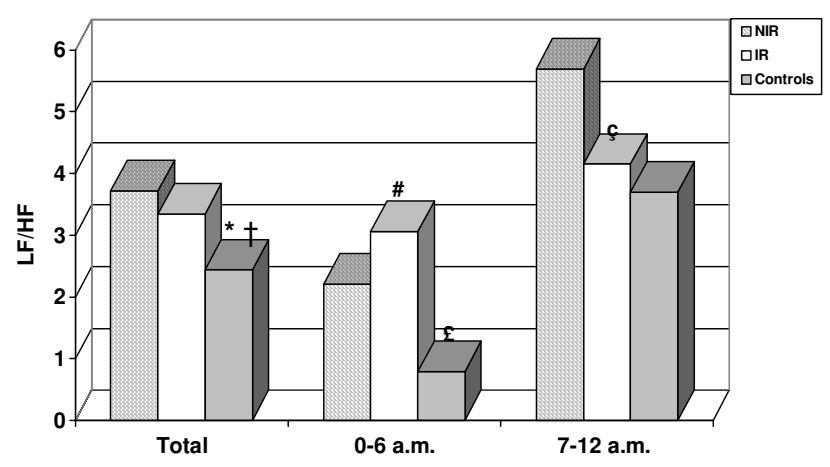

Figure 4

LF/HF value in offsprings of type 2 diabetic patients and control. IR: insulin resistant offsprings of type 2 diabetic patients, NIR: non insulin resistant offsprings of type 2 diabetic patients, LF/HF: low frequency/high frequency, hours of Holter registration: $0-6 \mathrm{am}=$ night time, $7-12 \mathrm{am}=$ morning. *p.0.016 controls vs IR; †p:0.002 control vs NIR; \# p:0.0I5 IR vs NIR; $\_$p:0.000I controls vs NIR and IR; ç p:0.043 controls vs NIR.

independently of insulin resistance. In frequency domain, the analysis of sympathetic (LF) and parasympathetic (HF) component and the symphatovagal balance (LF/HF) evidenced an association between the familiarity and a sympathetic overactivity, especially in nocturnal period, demonstrated by increase of LF (figure 2 and figure 3 ) and $\mathrm{LF} / \mathrm{HF}$ ratio (figure 4). This autonomic impairment is major in insulin resistant offsprings than non insulin resistant offsprings of type 2 diabetic patients. Moreover, our study had demonstrated, in time domain analysis of HRV, a significant reduction of the total autonomic system activity in both groups, expressed by progressive decrease of SDNN value from NIR to IR groups. These results indicated that the familiarity of type 2 diabetes mellitus is related to a global reduction of autonomic nervous system and that the dysautonomia increases if offsprings are insulin resistant.

In summary, a global reduction and alteration of circadian rhythm of autonomic activity are present in offspring of type 2 diabetic patients, without and with insulin resistance (figure 2,3 ).

A long term observation can answer the question whether autonomic abnormality precede the occurence of insuline resistence and play a role in the complex pathogenesis of the insulin resistance and type 2 diabetes mellitus. Others studies occurs to explain a possible pathogenetic role of autonomic dysfunction in the development of insulin resistance and type 2 diabetes mellitus. 


\section{Limitations}

A limitation in this study is the use of the HOMA index as a conventional indicator of insulin resistance. The best method for assessment of insulin resistance is the glucose clamp technique, however, the HOMA model has proved be a robust clinical and epidemiological tool in descriptions of the pathophysiology of diabetes, already quoted in $>500$ publications, it has become one of standard tools in the armamentarium of the clinical physiologist [23].

Other study are necessary to determine the mechanism whereby insulin-resistance may be related to autonomic dysfunction.

\section{List of abbreviations}

BMI: body mass index; DM: type 2 diabetes mellitus; HF: high frequency; HOMA-I: the homeostasis model assessment-index; HRV: heart rate variability; LF: low frequency; OGTTs: oral glucose tolerance tests; RMS-SD: the square root of the mean of the sum of the squares of differences between adjacent NN intervals; SDNN: The standard deviation of normal-to-normal RR intervals; WHR: waist-to-hip ratio.

\section{Competing interests}

The author(s) declare that they have no competing interests.

\section{References}

I. Pagani M, Lombardi F, Guzzetti S, Rimoldi O, Furlan R, Pizzinelli P, Sandrone G, Malfatto G, Dell'Orto S, Piccaluga E, et al.: Power spectral analysis of heart rate and arterial pressure variabilities as a marker of sympatho-vagal interaction in man and conscious dog. Circ Res 1986, 59(2): I78-93.

2. Malliani A, Pagani M, Lombardi F, Cerutti S: Cardiovascular neural regulation explored in the frequency domain. Circulation 1991, 84(2):482-92.

3. Montano N, Ruscone TG, Porta A, Lombardi F, Pagani M, Malliani A: Power spectrum analysis of heart rate variability to assess the changes in sympathovagal balance during graded orthostatic tilt. Circulation 1994, 90(4): |826-31.

4. Schwartz P, La Rovere M, Vanoli E: Autonomic nervous system and sudden cardiac death: experimental basis and clinical observations for post-myocardial infarction risk stratification. Circulation |992, 85:|77-|9|.

5. Malliani A, Schwartz $P$, Zanchetti A: Neural mechanism in life threatening arrhythmias. Am Heart J 1980, 200:705-7I5.

6. Tubani L, Baratta L, Giorgino F, Delfino M, Fiore G, Golluscio V, Giacovazzo $M$ : Heart rate variability in cluster headache. Ann Ital Med Int 2003, I 8(I):42-46.

7. Baratta L, Delfino M, Fiorentini A, Martuscelli M, Tubani L: Alterations of sympathovagal bilance by heart rate variability: a rare case of adult Still's disease. Ann ltal Med Int 2004, 19(3): 193-197.

8. Mastrocola C, Vanacore N, Giovani A, Locuratolo N, Vella C, Alessandri A, Baratta L, Tubani L, Meco G: Twenty-four-hour heart rate variability to assess autonomic function in Parkinson's disease. Acta Neurol Scand 1999, 99(4):245-247.

9. Laganà B, Tubani L, Maffeo N, Vella C, Makk E, Baratta L, Bonomo L: Heart rate variability and cardiac autonomic function systemic lupus erythematosus. Lupus 1996, 5(I):49-55.

10. Ewing DJ, Neilson JM, Shapiro CM, Stewart JA, Reid W: Twenty four hour heart rate variability: effects of posture, sleep, and time of day in healthy controls and comparison with bedside tests of autonomic function in diabetic patients. Br Heart $\int 1991$, 65:239-244.

II. Ziegler D: Diabetic cardiovascular autonomic neuropathy: prognosis, diagnosis and treatment. Diabetes Metab Rev 1994, 10:339-383.

12. Malpas SC, Maling TJB: Heart rate variability and cardiac autonomic function in diabetes. Diabetes 1990, 39:। I77-I I8I

13. Pagani M, Malfatto G, Pierini S, Casati R, Masu AM, Poli M, Guzzetti S, Lombardi $F$, Cerutti $S$, Malliani A: Spectral analysis of heart rate variability in the assessment of autonomic diabetic neuropathy. J Auton Nerv Syst 1988, 23:143-I5.

14. Bianchi A, Bontempi B, Cerutti S, Gianoglio P, Comi G, Natali Sora MG: Spectral analysis of heart rate variability signal and respiration in diabetic subjects. Med Biol Eng Comput 1990, 28:205-2II.

15. Bellavere F, Balzani I, De Masi G, Carraro M, Carenza P, Cobelli C, Thomaseth K: Power spectral analysis of heart rate variation improves assessment of diabetic cardiac autonomic neuropathy. Diabetes 1992, 41:633-640.

16. Van den Akker T], Koelman ASM, Hogenhuis LAH, Rompelman G: Heart rate variability and blood pressure oscillations in diabetics with autonomic neuropathy. Automedica 1983, 4:201-208.

17. Brunner EJ, Hemingway $H$, Walker BR, Page $M$, Clarke P, Juneja $M$, Shipley MJ, Kumari M, Andrew R, Seckl JR, Papadopoulos A, Checkley S, Rumley A, Lowe GD, Stansfeld SA, Marmot MG: Adrenocortical, autonomic, and inflammatory causes of the metabolic syndrome: nested case-control study. Circulation 2002, I 06(2I):2659-65.

18. Pikkujamsa SM, Huikuri HV, Airaksinen KE, Rantala AO, Kauma H, Lilja M, Savolainen MJ, Kesaniemi YA: Heart rate variability and baroreflex sensitivity in hypertensive subjects with and without metabolic features of insulin resistance syndrome. $\mathrm{Am} J$ Hypertens 1998, I I(5):523-3I.

19. Flanagan DE, Vaile JC, Petley GW, Moore VM, Godsland IF, Cockington RA, Robinson JS, Phillips DI: The autonomic control of heart rate and insulin resistance in young adults. J Clin Endocrinol Metab 1999, 84(4): I 263-7.

20. Frontoni S, Bracaglia D, Baroni A, Pellegrini F, Perna M, Cicconetti E, Ciampittiello G, Menzinger G, Gambardella S: Early autonomic dysfunction in glucose-tolerant but insulin-resistant offspring of type 2 diabetic patients. Hypertension 2003, 4 I (6): | 223-7.

21. De Angelis C, Perelli P, Trezza R, Casagrande M, Biselli R, Pannitteri $G$, Marino B, Farrace S: Modified autonomic bilance in offsprings of diabetics detected by spectral analysis of heart rate variabilità. Metabolism 200 I, 50(I I): I 270-74.

22. Laitinen T, Vauhkonen IK, Niskanen LK, Hartikainen JE, Lansimies EA, Uusitupa MI, Laakso M: Power spectral analysis of heart rate variability during hyperinsulinemia in nondiabetic offspring of type 2 diabetic patients: evidence for possible early autonomic dysfunction in insulin-resistant subjects. Diabetes 48(6): I 295-9.

23. European Society of Hypertension-European Society of Cardiology Guidelines Committee: Guidelines for the management of arterial hypertension. J Hypertens 2003, 2 I(6): I0 I I-53.

24. American Diabetes Association: Diagnosis and classification of diabetes mellitus. Diabetes Care 2004, 27(SuppI I):S5-SIO.

25. Albareda M, Rodriguez-Espinosa J, Murugo M, de Leiva A, Corcoy R: Assessment of insulin sensitivity and beta-cell function from measurements in the fasting state and during an oral glucose tolerance test. Diabetologia 2000, 43( I 2): I 507-I I.

26. Wallace TM, Levy JC, Matthews DR: Use and abuse of HOMA modeling. Diabetes Care 2004, 27(6): I 487-95.

27. Katsuki A, Sumida Y, Gabazza EC, Murashima S, Furuta M, Araki-Sasaki R, Hori $Y$, Yano $Y$, Adachi $Y$ : Homeostasis model assessment is a reliable indicator of insulin resistance during follow-up of patients with type 2 diabetes. Diabetes Care 200I, 24(2):362-5.

28. Bonora E, Targher G, Alberiche M, Bonadonna RC, Saggiani F, Zenere $\mathrm{MB}$, Monauni T, Muggeo M: Homeostasis model assessment closely mirrors the glucose clamp technique in the assessment of insulin sensitivity: studies in subjects with various degrees of glucose tolerance and insulin sensitivity. Diabetes Care 2000, 23(I):57-63.

29. Matthews DR, Hosker JP, Rudenski AS, Naylor BA, Treacher DF, Turner RC: Homeostasis model assessment: insulin resistance 
and beta-cell function from fasting plasma glucose and insulin concentrations in man. Diabetologia 1985, 28(7):412-9.

30. Task Force of the European Society of Cardiology and the North American Society of Pacing and Electrophysiology: Heart rate variability: standards of measurement, physiological interpretation and clinical use. Circulation 1996, 93(5): 1043-65.

Publish with Bio Med Central and every scientist can read your work free of charge

"BioMed Central will be the most significant development for disseminating the results of biomedical research in our lifetime. " Sir Paul Nurse, Cancer Research UK

Your research papers will be:

- available free of charge to the entire biomedical community

- peer reviewed and published immediately upon acceptance

- cited in PubMed and archived on PubMed Central

- yours - you keep the copyright

Submit your manuscript here:

http://www.biomedcentral.com/info/publishing_adv.asp 